\title{
review
}

\section{Treatment-related cardiovascular toxicity in long-term survivors of testicular cancer}

\author{
Jasenka Gugic, Lorna Zadravec Zaletel, Irena Oblak \\ Department of Radiotherapy, Institute of Oncology Ljubljana, Ljubljana, Slovenia
}

Radiol Oncol 2017; 51(2): 221-227.

Received 4 February 2016

Accepted 14 February 2016

Correspondence to: Lorna Zadravec Zaletel, M.D., Ph.D., Department of Radiotherapy, Institute of Oncology Ljubljana, Zaloška 2, SI-1000 Ljubljana, Slovenia. Phone: +386 15879 500. E-mail: Izaletel@onko-i.si

Disclosure: No potential conflicts of interest were disclosed.

\begin{abstract}
Backgrounds. Testicular cancer is the most common malignancy in young men. Considering increasing incidence, exceptionally high cure rate, as well as long life expectancy, assessment of long term toxicity in testicular cancer survivors is of great importance. In the last decades a major effort has been made in order to reduce toxicity of treatment, while maintaining its high effectiveness.

Conclusions. Actual knowledge on treatment toxicity is based on outdated treatment modalities. Hopefully, modern treatment modalities could reduce toxicity, but, there is no firm confirmation for that at the moment, as data dealing with late sequelae of modern treatment of testicular cancer are not available yet due to the short period of observation. The life-threatening cardiovascular toxicity in testicular cancer survivors is major complication of platinum-based chemotherapy, mediastinal radiotherapy and even subdiaphragmatic radiotherapy.
\end{abstract}

Key words: testicular cancer; cardiovascular toxicity; long-term survivors

\section{Introduction}

Although rare disease, testicular cancer is the most common malignancy in young men aged 20 to 40 years. Considering increasing incidence worldwide ${ }^{1-3}$, exceptionally high cure rate with 5-year survival rate exceeding $95 \%$ for all stages ${ }^{4}$ and life expectancy almost comparable to age-matched healthy male population ${ }^{5}$, evaluation of treatmentrelated long-term morbidity has become increasingly important. In the past decades efforts have been made in order to optimize treatment with objective to decrease toxicity, while maintaining high cure rates. The introduction of less toxic chemotherapeutic schemes ${ }^{6-8}$, reduction in radiation doses and volumes ${ }^{9-11}$ and preferred use of active surveillance $^{12}$, have all led to reduced treatment toxicity. Irrespective of that, some treatment-related sequelae remained unavoidable. Most of the current studies represent complications of treatment modalities administered several years or even decades ago, but are still of a major concern.
With the 25-year risk of approximately $16 \%$, cardiovascular diseases (CVD) are among the most important life-threatening long-term complications in testicular cancer survivors. ${ }^{13}$ Long-term testicular cancer survivors are more likely to develop unfavourable cardiovascular risk profile. ${ }^{14-21}$ In addition, an increased risk of mortality caused by CVD has also been observed.5,22-24

According to the current knowledge, cardiovascular toxicity is mainly due to cisplatin based chemotherapy and mediastinal radiation therapy ${ }^{13,19,25}$; although subdiaphragmatic radiotherapy is associated with an increased risk of CVD as well. ${ }^{14,26}$

\section{Treatment-related cardiovascular morbidity}

The introduction of cisplatin based chemotherapeutic regimens into treatment of testicular cancer in the early 1970s represents cornerstone in 
TABLE 1. Risk for coronary artery disease in testicular cancer survivors regarding to different treatment modalities

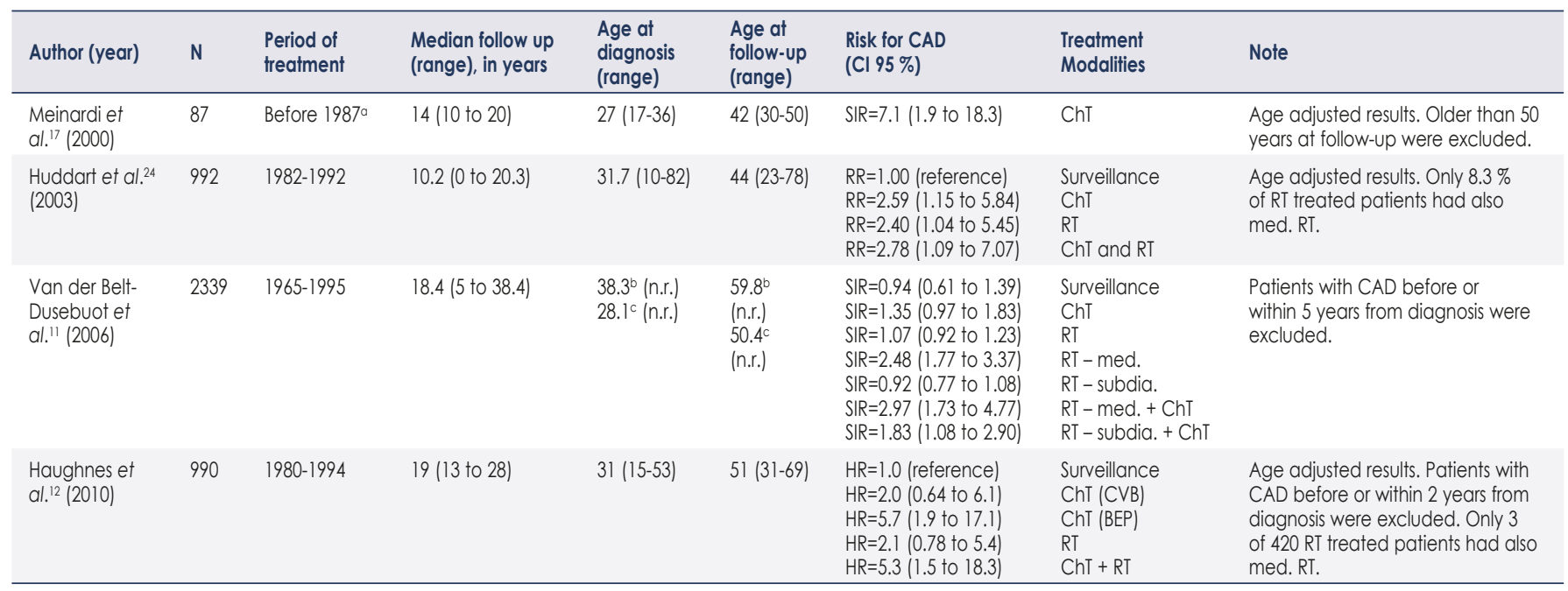

$\mathrm{BEP}=$ bleomycin, etoposid, cisplatin; $\mathrm{CAD}=$ coronary artery disease; $\mathrm{ChT}=$ chemotherapy; $\mathrm{CVB}=$ cisplatin, etoposid, bleomycin; $\mathrm{HR}=$ hazard ratio; med. = mediastinal; $\mathrm{N}=$ number of patients; n.r. = not reported; $\mathrm{RT}=$ radiotherapy; $\mathrm{RR}=$ relative risk; SIR = standardized incidence ratio; subdia. = subdiaphragmatic

a All patients treated with cisplatin based chemotherapy before 1987.

${ }^{b}$ Age for seminoma patients.

cAge for nonseminoma patients.

testicular cancer management. Prior to that, different chemotherapeutic regimens were used, some of them containing even anthracyclines, cytotoxic drugs with well known cardiotoxic effect. Although reports on cisplatin cardiovascular toxicity date back to the $1980 \mathrm{~s}^{20,27-29}$, first study systematically reporting the frequency of late cardiac morbidity by Meinardi et al. was published in 2000 . After a median follow up of 14 years, a major cardiac event was documented in 5 of 87 patients $(5.8 \%)$ treated with cisplatin based chemotherapy; myocardial infarction in $2(2.3 \%)$ and angina pectoris in the remaining $3(3.5 \%)$ patients. Compared with the general male population, this corresponded to approximately 7-fold increased risk (observed-toexpected ratio $[\mathrm{O} / \mathrm{E}]=7.1,95 \%$ confidence interval [CI], 1.9 to 18.3 -fold). In this study, additional analysis of subclinical cardiac disease was performed as well. Echocardiographic evaluation showed abnormal diastolic function of the left ventricle in $33 \%$ of the patients treated with cisplatin based chemotherapy, probably as an early sign of microvasculopathy. ${ }^{19}$

Subsequent studies with larger testicular cancer cohorts in following years revealed the magnitude of the problem (Table 1). Huddart et al. reported on cardiovascular morbidity in 992 testicular cancer survivors, treated between 1982 and 1992 at the Royal Marsden Health Service Trust. In particular, they reported on cardiac events, comprising angina pectoris, long-term chest pain, myocardial infarction, surgery for coronary artery disease and others cardiac abnormalities. Approximately two thirds of the patients were treated with chemotherapy; cisplatin-based in two thirds and carboplatinbased in remaining third. After a median follow up of 10.2 years, age-adjusted relative risk (RR) for cardiac events was 2.59 (95\% CI, 1.15 to 5.84) with no significant difference between patients treated with cisplatin and those treated with carboplatin. There was no difference in risk for cardiac events between patients treated with bleomycin containing regimens and those, who didn't get bleomycin, indicating that bleomycin did not significantly contribute to cardiac damage. Moreover, in this study, risk for cardiac events was increased in patients treated with radiotherapy $(\mathrm{RR}=2.4,95 \% \mathrm{CI}, 1.04$ to 5.45$)$, although a minority of them - only $8.3 \%$, received mediastinal radiotherapy. Patients treated with chemotherapy and radiotherapy were, as expected, at the highest risk, with age-adjusted $\mathrm{RR}=$ 2.78 (95\% CI, 1.09 to 7.07$){ }^{26}$

After a median follow up of 18.4 years, van der Belt-Dusebout et al. reported on 694 cardiovascular events in 2512 5-year testicular cancer survivors. The overall standardized incidence ratio (SIR) for coronary artery disease was significantly elevated $(\mathrm{SIR}=1.17,95 \% \mathrm{CI}, 1.04$ to 1.31$)$. They found slightly, but not significantly increased risk for coronary artery disease in chemotherapy treated patients 
compared to the general population (SIR $=1.35$, $95 \% \mathrm{CI}, 0.97$ to 1.83$)$. Chemotherapy with cisplatin, vinblastine, bleomycin (PVB) regimen was associated with 1.9-fold (95\% CI, 1.7 to 2.0-fold), significantly increased risk for myocardial infarction, while chemotherapy with bleomycin, etoposide, cisplatin (BEP) was associated with borderline significantly increased risk for CVD (SIR $=1.5,95 \%$ CI, 1.0 to 2.2), but not for myocardial infarction. Analysis of patients treated with radiotherapy revealed 3.7-fold ( $95 \%$ CI, 2.2 to 6.2-fold) increased risk for myocardial infarction after a mediastinal radiotherapy, while there was no increased risk for CVD after subdiaphragmatic radiotherapy. There was a trend towards higher risk for coronary artery disease with younger age at diagnosis and younger age at follow up as well, for the whole study group. In addition, in nonseminoma group of patients there was a significant influence of attained age of patient on the risk for myocardial infarction, with increased risk in 54 years old or younger (SIR $=1.86,95 \% \mathrm{CI}, 1.20$ to 2.74 ), even higher risk in younger than 45 years (SIR $=2.06,95 \% \mathrm{CI}, 1.15$ to 3.41 ) and decreased risk in 55 years or older $(\mathrm{SIR}=$ $0.53,95 \%$ CI, 0.25 to 0.98$){ }^{13}$

Haugnes et al. recently reported on cardiovascular morbidity in 990 testicular cancer survivors treated between 1980 and 1994. The incidence of two end-points were evaluated: coronary artery disease, including myocardial infarction and angina pectoris and atherosclerotic diseases, including coronary artery disease, cerebrovascular insult, transitory ischemic attack, carotid stenosis and other peripheral atherosclerotic disease. With a median follow up of 19 years, testicular cancer survivors treated with chemotherapy had increased risk for coronary artery disease (hazard ratio $[\mathrm{HR}]=2.6$, $95 \% \mathrm{CI}, 0.96$ to 6.9 ) and atherosclerotic disease (HR $=2.6,95 \% \mathrm{CI}, 1.1$ to 5.9 ), compared to surgery only group. Treatment with BEP was associated with a 5.7-fold (95\% CI, 1.9 to 17.1-fold), significantly increased risk for coronary artery disease and a 4.7-fold (95\% CI, 1.8 to 12.2 -fold), significantly increased risk for atherosclerotic disease, while treatment with CVB was associated with nonsignificantly increased risk for CVD, in contrary with the results of van der Belt-Dusebout study. Additional subanalyses, taking into account chemotherapy type, revealed that higher cumulative dose of etoposide was associated with increased risk for coronary artery disease, while higher cumulative doses of etoposide and cisplatin were associated with increased risk for atherosclerotic disease as well. Interestingly, risk for coronary artery disease and atherosclerotic disease were increased even in patients treated with mostly non-mediastinal radiotherapy (only 3 of 420 irradiated patients received mediastinal radiotherapy), being 2.1-fold (95\% CI, 0.78 to 5.4 -fold) and 2.3 -fold ( $95 \%$ CI, 1.03 to 5.3-fold), respectively. In accordance with results of other studies, treatment with chemotherapy and radiotherapy posed the highest risk, 5.3-fold (95\% CI, 1.5 to 18.3 -fold) for coronary artery disease and 4.7-fold (95\% CI, 1.6 to 14.1-fold) for atherosclerotic disease, respectively. ${ }^{14}$

\section{Treatment-related cardiovascular mortality}

Hanks et al. reported on elevated cardiac mortality after 15-years follow up in 387 testicular cancer survivors treated with radiotherapy in 1973 and 1974. Seventy-nine percent of patients with stage II and $27 \%$ of patients with stage I had mediastinal irradiation. A significant, 3.1-fold increase in noncancer mortality was observed compared to general male population. Further analysis of causes of death revealed a 2.3-fold increase in cardiac deaths. Eight out of ten patients, who died due to cardiac disease, received mediastinal irradiation. ${ }^{22}$

In Zagars' study only minority of patients (71 of 477) received mediastinal irradiation. The cardiac mortality rate was significantly elevated only beyond 15 years of follow-up with standardized mortality ratio (SMR) 1.95 (95\% CI, 1.24 to 2.94). Cardiac mortality rate was not significantly elevated during the first 15 years of follow up for the whole group of patients, except for the subgroup of patients treated with mediastinal irradiation (SMR $=1.63,95 \%$ CI, 0.44 to 4.17$){ }^{23}$

Fossa et al. was the first author reporting on cardiac mortality among testicular cancer survivors treated with combination of radiotherapy and chemotherapy. They found slightly, but significantly increased risk of dying from circulatory disease (SMR $=1.20,95 \% \mathrm{CI}, 1.0$ to 1.5). A comparison according to the diagnostic periods failed to show increase of mortality after the introduction of cisplatin, probably due to other treatment modifications that happened in that period: omission of mediastinal irradiation, confinement of subdiaphragmatic fields and introduction of modern radiotherapy planning and delivery facilities. ${ }^{24}$

In another large international study by Fossa et al., there was no significant increase in overall mortality from circulatory disease in whole study population of 38907 patients treated for testicular cancer with only surgery, chemotherapy or ra- 
diotherapy or with combination of chemotherapy and radiotherapy. However, a significantly increased mortality due to hypertensive disorder (SMR $=1.39,95 \%$ CI, 1.01 to 1.89$)$ was documented. Mortality caused by all circulatory diseases was significantly higher in testicular cancer survivors treated with chemotherapy $(\mathrm{SMR}=1.44,95 \% \mathrm{CI}$, 1.06 to 1.91) compared to group of patients treated with radiotherapy or surgery only. The risk was even higher in testicular cancer survivors treated with radiotherapy and chemotherapy $(\mathrm{SMR}=2.06$, $95 \% \mathrm{CI}, 1.27$ to 3.14 ). In comparision with the general population, mortality from circulatory disease was significantly increased in patients treated with radiotherapy at the age under 35 years $(\mathrm{SMR}=1.7$, $95 \% \mathrm{CI}, 1.21$ to 2.31 ), as well as in patients treated with chemotherapy (with or without radiotherapy) at the age under 35 years after $1975(\mathrm{SMR}=1.58$, $95 \%$ CI, 1.25 to 2.01$)$. The latter coincides with introduction of cisplatin based chemotherapy. ${ }^{5}$

\section{Pathophysiology of CVD caused by cisplatin based chemotherapy}

Although mechanisms that would explain an increased risk of CVD after cisplatin based chemotherapy are not completely clarified, endothelial damage is considered to play a main role in pathogenesis. Microalbuminuria, thought to be an early sign of endothelial damage, was reported by Meinardi et al. in $22 \%$ of patients treated with cisplatin based chemotherapy. ${ }^{19}$ Patients with microalbuminuria had a tendency of higher blood pressure, which could be a consequence of systemic endothelial damage as well. ${ }^{30}$ Moreover, microalbuminuria was shown to be a predictor of cardiac events. ${ }^{31,32}$ Nuver et al. observed microalbuminuria in $12 \%$ of patients treated with cisplatin based chemotherapy for testicular cancer after median follow up of 7 years, compared to $0 \%$ in surgery only group and $4.6 \%$ in a larger group of healthy men from general population. ${ }^{33}$

Nuver et al. proposed other endothelial and inflammatory markers as potential early indicators of endothelial damage and atherosclerosis, which is essentially an inflammatory proces. ${ }^{33}$ Namely, they found significantly higher levels of von Willebrandt factor (vWF), tissue plasminogen activator (t-PA), plasminogen activator inhibitor-1 (PAI-1), fibrinogen and high sensitivity C-reactive protein (hs-CRP) in patients treated with chemotherapy compared to healthy men. Elevated levels of endothelial and inflammatory markers were shown to be associated with higher risk for coronary artery disease. ${ }^{34,35}$ In addition, elevated levels of inflammatory proteins were associated with more pronounced atherosclerosis as well. ${ }^{36}$ Moreover, according to the available data from the literature, hs-CRP could be a predictive marker for CVD. ${ }^{36-39}$ In the same study by Nuver et al. evaluation of vascular structure and function of common carotid artery was performed. Irrespective of high levels of endothelial and inflammatory markers, this evaluation failed to show any significant changes in wall structure or function of blood vessel, except a small, but statistically significant increase in carotid wall stiffness. Explanation for this could be impairment of microvasculature at the early stages, with later impairment of the macrovasculature. The same author in another study also reported on small, but statistically significant increase in carotid intima media thickness in patients treated with cisplatin based chemotherapy, which was shown to be associated with higher myocardial infarction incidence. ${ }^{40}$

Gietema et al. showed a presence of circulating platinum in plasma up to 20 years after treatment with cisplatin based chemotherapy in testicular cancer survivors, which, together with the awareness of signs for endothelial damage, led to the theory that cisplatin may chronically stimulate the endothelium, eventually resulting in vasculature impairment. ${ }^{41,42}$ Correlation between cisplatin plasma levels and severity of neurotoxicity was recently shown, indicating that cisplatin level may serve as a putative marker for long-term toxicity, perhaps even for CVD. ${ }^{43}$

\section{Unfavourable cardiovascular risk profile, metabolic syndrome and hypogonadism in testicular cancer survivors}

CVD following cisplatin based chemotherapy could, on the other hand, be caused by a gradual development of unfavourable cardiovascular risk profile. Namely, several studies reported significantly higher prevalence of hypertension in testicular cancer survivors treated with platinum based chemotherapy. ${ }^{14,16,19}$ Another cause of hypertension in testicular cancer survivors could be abdominal radiotherapy. Namely, some studies with patients treated with abdominal radiotherapy for malignant disease in abdomen reported on increased risk of hypertension. Possible explanation for this is development of radiation nephropathy with resulting hypertension, probably through a renovascular mechanism with activation of the reninangiotensin-aldosteron system. ${ }^{44-46}$ Furthermore, a 
few studies reported on increased risk for hypercholesterolemia, as well as increased prevalence of obesity following cisplatin based chemotherapy in testicular cancer survivors, which was, on the other hand, not confirmed by others. ${ }^{14-19,21}$ Haugnes et al. found significantly higher levels of $\mathrm{HbA1c}$ in all chemotherapy and radiotherapy treated testicular cancer patients compared to surgery only group, moreover, patients treated with radiotherapy were at a greater risk of being diagnosed with diabetes as well. Namely, the prevalence of diabetes for the whole study group was $7.3 \%$, while it was $10.2 \%$ and $15.6 \%$ for radiotherapy and radiotherapy/ chemotherapy group, respectively. The most likely explanation for this is radiation damage of pancreatic gland tissue, as the large part of the gland is included in subdiaphragmatic radiation field. Insulin resistance in testicular cancer patients treated with chemotherapy could be a consequence of persistent hypomagnesemia, caused by cisplatininduced damage of proximal renal tubules. In Haugnes' study it was found that the prevalence of other unfavourable risk factors for CVD was the highest in radiotherapy/chemotherapy group as well, suggesting a possibility of synergistic effect. ${ }^{14}$

Hypertension, dyslipidaemia, obesity and insulin resistance are all components of the metabolic syndrome. Considering that testicular cancer survivors are at greater risk to have one or more of these components, the metabolic syndrome could be a possible causal link between cytotoxic treatment and CVD. ${ }^{47,48}$ The prevalence rates of metabolic syndrome in testicular cancer survivors range from $8 \%$ to $40 \%$, depending on applied criteria for diagnosis of this syndrome. Patients treated with chemotherapy are at higher risk for development of metabolic syndrome, compared to controls and to radiotherapy or surgery only group. This is especially true for patients receiving higher cumulative dose of cisplatin - $850 \mathrm{mg}$ or more. Beside association with cumulative dose of cisplatin, a positively correlation was demonstrated between prevalence of metabolic syndrome and cumulative doses of bleomycin and etoposide as well. ${ }^{48}$ The aetiology of the metabolic syndrome is not entirely clear. Gietema et al. were the first authors reporting on the possible association of metabolic syndrome with low serum testosterone levels in patients who received chemotherapy. ${ }^{49}$ Nuver et al. proposed that low serum testosterone level and metabolic syndrome could be associated through increased body mass index. ${ }^{48}$

It's well known that hypogonadism increases risk for CVD and correlates with the severity of ath- erosclerosis, even in a healthy male population. In addition, it was shown that gonadal dysfunction is associated with obesity, dyslipidaemia and insulin resistance, as well as with higher levels of markers of endothelial damage. A middle aged men from general population with free testosterone levels in the lower third of normal range were at a 2.7-fold (95\% CI, 2.0 to 3.7-fold) increased risk for metabolic syndrome in age-adjusted analyses. After further adjusting for body mass index, low free testosterone level was associated with 1.7-fold (95\% CI, 1.2 to 2.4-fold) increased risk for metabolic syndrome. This correlation was even more pronounced for total testosterone levels and sex-hormone binding globulin levels. ${ }^{50}$ In testicular cancer survivors, gonadal dysfunction may be result of prior treatment with chemotherapy, subdiaphragmatic radiotherapy and with surgery as well. ${ }^{51}$

However, it is still ongoing debate, whether metabolic syndrome is a consequence of hypogonadism or intrinsic feature of testicular cancer survivors, as a part of testicular dysgenesis syndrome. If the first assumption is true, the testosterone substitution therapy could have a favourable impact on metabolic syndrome and development of CVD in testicular cancer survivors. To date, there is no evidence for that. According to the literature, substitution therapy with testosterone might be useful in men with significantly reduced testosterone levels, while its benefit is questionable in a case of modest hypogonadism. ${ }^{48}$

\section{Pathophysiology of CVD caused by irradiation}

Increased risk for CVD after mediastinal radiotherapy in patients treated for testicular cancer is a consequence of a direct exposure of the heart to irradiation. ${ }^{52-54}$ Data from the literature indicate that irradiation of the heart leads to the tissue damage through microvasculopathy and eventually macrovasculopathy. Endothelial damage of small blood vessels is due to radiation induced generation of reactive oxygen species. Typically, irregularities of the endothelial cell membranes, cytoplasmic swelling, thrombosis and rupture of the walls are present in early phase. This eventually results in reduced ratio of capillaries to myocytes by approximately $50 \%$, leading to ischaemia and fibrosis in late phase. ${ }^{55}$

Less is known about pathologic association between subdiaphragmatic radiotherapy and CVD. According to the physical model, in series of Huddart et al., the expected mean dose to the 
heart in a case of "dog leg" radiation field, extending upwards to the bottom of tenth thoracic vertebra, was approximately $2.5 \%$ of the total dose, which is unlikely to cause any serious damage. ${ }^{26}$ Possible mechanism of CVD in patients treated with abdominal radiotherapy could be radiation nephropathy, as well as hypogonadism, as mentioned above. A direct endothelial damage, eventually resulting in atherosclerosis, is another possible mechanism, supported by increased levels of hs-CRP in patients treated with radiotherapy. ${ }^{56}$

Up to 1980 's, total dose of subdiaphragmatic radiotherapy was 36 to $40 \mathrm{~Gy}$ and even more. After that, radiation dose was gradually reduced, being 20 Gy for stage I seminoma, 30 Gy for stage II A and 36 Gy for stage II B. In case of prophylactic mediastinal irradiation, testicular cancer survivors usually received 30 Gy. Fractionation, mainly used in subdiaphragmatic, as well as in mediastinal irradiation, is 2 Gy daily fractions 5 days per week.

\section{Implication for future}

Current knowledge about long-term treatment-related toxicity in testicular cancer survivors is based on treatment modalities administered years to decades ago. Optimization of treatment, including omission of mediastinal irradiation, confinement of subdiaphragmatic fields, use of modern radiotherapy planning and delivery techniques, lowering the cumulative doses of cytotoxic drugs and preferential use of active surveillance will probably all reduce risk for cardiac toxicity. ${ }^{7-12}$ However, as data from newer studies are not yet available, we do not have confirmation for this and further research is essential. ${ }^{57}$

Following successful treatment, most of the testicular cancer survivors are under the medical surveillance of their oncologists for subsequent 5 to 10 years. Obviously, these patients need a longer, probably life-long follow-up with special attention to modifiable CVD risk factors. Patients should be treated for hypertension, diabetes, hyperlipidaemia and dyslipidaemia and advised about healthy lifestyle. A smoking cessation is highly recommended, along with regular physical activity and maintaining of optimal body weight. At the moment, no guidelines concerning CVD risk in testicular cancer survivors are available and several study groups proposed their development. ${ }^{25}$

\section{References}

1. Znaor A, Lortet-Tieulent J, Jemal A, Bray F. International variations and trends in testicular cancer incidence and mortality. Eur Urol 2014; 65: 1095-106.

2. Trabert B, Chen J, Devesa SS, Bray F, McGlynn KA. International patterns and trends in testicular cancer incidence, overall and by histologic subtype, 1973-2007. Andrology 2015; 3: 4-12.

3. Kovač V. The cause of testicular cancer. Radiol Oncol 1998; 32: 201-5.

4. National Cancer Institute. Surveillance, Epidemiology, and End Results (SEER) Program. [citated 2 Feb 2016]. Available at https://seer.cancer.gov/ statfacts/htlm/testis.ht/m

5. Fossa SD, Gilbert E, Dores GM, Chen J, McGlynn KA, Schonfeld S, et al.. Noncancer causes of death in survivors of testicular cancer. $J$ Natl Cancer Inst 2007; 99: 533-44.

6. De Wit R, Roberts JT, Wilkinson PM, de Mulder PHM, Mead GM, Fossa SD, et al. Equivalence of three or four cycles of bleomycin, etoposide, and cisplatin chemotherapy and of a 3- or 5-day schedule in good-prognosis germ cell cancer: a randomized study of the European EORTC-GTCCG and. J Clin Oncol 2001; 19: 1629-40.

7. Saxman SB, Finch D, Gonin R, Einhorn LH. Long-term follow up of a phase III study of three versus four cycles of bleomycin, etoposide, and cisplatin in favorable-prognosis germ-cell tumors: The Indiana University experience. $J$ Clin Oncol 1998; 16: 702-6

8. Nichols CR, Wiliams SD, Loehrer PJ, Greco FA, Crawford ED, Weetlaufer J, et al. Randomized study of cisplatin dose intensity in poor-risk germ cell tumors: A Southeastern Cancer Study Group and Southwest Oncology Group Protocol. J Clin Oncol 1991; 9: 1163-72.

9. Fossa SD, Horwich A, Russell JM, Roberts JT, Cullen MH, Hodson NJ, et al. Optimal planning target volume for stage I testicular seminoma: A MRC randomized trial. J Clin Oncol 1999; 17: 1146.

10. Jones WG, Fossa SD, Mead GM, Roberts JT, Sokal M, Horwich A, et al. Randomized trial of 30 versus $20 \mathrm{~Gy}$ in the adjuvant treatment of stage seminoma: A report on MRC Trial TE 18, EORTC Trial 30942. J Clin Oncol 2005; 23: 1200-8.

11. Kovač V. Prevention of fertility disturbances in oncological male patients. Radiol Oncol 1996; 30: 286-90.

12. Horwich A, Alsanjari N, A'Hern R, Nicholls J, Dearnaley DP, Fisher C Surveillance following orchidectomy for stage I testicular seminoma. $\mathrm{Br} J$ Cancer 1992; 65: 775-8.

13. Van den Belt-Dusebout AW, Nuver J, de Wit R, Gietema JA, ten Bokke Huinink WW, Rodrigus PT, et al. Long-term risk of cardiovascular disease in 5-year survivors of testicular cancer. J Clin Oncol 2006; 24: 467-75.

14. Haugnes HS, Wethal T, Aass N, Dahl O, Klepp O, Langberg CW, et al. Cardiovascular risk factors and morbidity in long-term survivors of testicular cancer: A 20-year follow-up study. J Clin Oncol 2010; 28: 4649-57.

15. Raghavan D, Cox K, Childs A, Grygiel J, Sullivan D. Hypercholesterolemia after chemotherapy for testis cancer. J Clin Oncol 1992; 10: 1386-9.

16. Sagstuen H, Aass N, Fossa SD, Dahl O, Klepp O, Wist EA, et al. Blood pressure and body mass index in long-term survivors of testicular cancer. J Clin Oncol 2005; 23: 4980-90.

17. Nord C, Fossa SD, Egeland T. Excessive annual BMI increase after chemotherapy among young survivors of testicular cancer. Br J Cancer 2003; 88: 36-41.

18. Strumberg D, Brugge S, Korn MW, Koeppen S, Ranft J, Scheiber G, et al. Evaluation of long-term toxicity in patients after cisplatin-based chemotherapy for non-seminomatous testicular cancer. Ann Oncol 2002; 13: 229-36.

19. Meinardi MT, Gietema JA, van der Graaf WTA, van Veldhuisen DJ, Runne MA, Sluiter WJ, et al. Cardiovascular morbidity in long-term survivors of metastatic testicular cancer. J Clin Oncol 2000; 18: 1725-32.

20. Stoter G, Koopman A, Vendrik CP, Struyvenberg A, Sleyfer DT, Willemse PH et al. Ten-year survival and late sequelae in testicular cancer patients treated with cisplatin, vinblastine, and bleomycin. J Clin Oncol 1989; 7: 1099-104. 
21. Gietema JA, Sleijfer DT, Willemse PH, Schraffordt HK, van Ittersum E, Verschuren WM, et al. Long-term follow-up of cardiovascular risk factors in patients given chemotherapy for disseminated nonseminomatous testicular cancer. Ann Intern Med 1992; 116: 709-15.

22. Hanks GE, Peters T, Owen J. Seminoma of the testis: long-term beneficial and deleterious results of radiation. Int J Radiat Oncol Biol Phys 1992; 24: 913-19.

23. Zagars GK, Ballo MT, Lee AK, Strom SS. Mortality after cure of testicular seminoma. J Clin Oncol 2004; 22: 640-7.

24. Fossa SD, Aass N, Harvei S, Tretli S. Increased mortality rates in young and middle-aged patients with malignant germ cell tumours. Br J Cancer 2004; 90: 607-12.

25. Van den Belt-Dusebout AW, de Wit R, Gietema JA, Horenblas S, Louwmann MWJ, Ribot JG, et al. Treatment-specific risk of second malignancies and cardiovascular disease in 5-year survivors of testicular cancer. $J$ Clin Oncol 2007; 25: 4370-8.

26. Huddart RA, Norman A, Shahidi M, Horwich A, Coward D, Nicholls J, et al. Cardiovascular disease as a long-term complication of treatment for testicular cancer. J Clin Oncol 2003; 21: 1513-23.

27. Samuels BL, Vogelzang NJ, Kennedy BJ. Severe vascular toxicity associated with vinblastine, bleomycin, and cisplatin chemotherapy. Cancer Chemother Pharmacol 1987; 19: 253-6.

28. Lederman GS, Sheldon TA, Chaffey JT, Herman TS, Gelman RS, Coleman CN. Cardiac disease after mediastinal irradiation for seminoma. Cancer 1987; 60: 772-6.

29. Bokemeyer C, Berger CC, Kuczyk MA, Schmoll HJ. Evaluation of long-term toxicity after chemotherapy for testicular cancer. J Clin Oncol 1996; 14: 2923-32.

30. Pedrinelli R, Giampietro O, Carmassi F, Melillo E, Dell'Omo G, Catapano G, et al. Microalbuminuria and endothelial dysfunction in essential hypertension. Lancet 1994; 344: 14-18.

31. Stehouwer CD, Yudkin JS, Fioretto P, Nosadini R. How heterogeneous is microalbuminuria in diabetes mellitus? The case for "benign " and "malignant microalbuminuria. Nephrol Dial Transplant 1998; 13: 2751-4.

32. Hillege HL, Fidler V, Diercks GF, van Gilst WH, de Zeeuw D, van Veldhuisen DJ, et al. Urinary albumin excretion predicts cardiovascular and noncardiovascular mortality in general population. Circulation 2002; 106: 1777-82.

33. Nuver J, Smit AJ, Sleijfer DT, van Gessel Al, van Roon AM, van der Meer J, et al. Microalbuminuria, decreased fibrinolysis, and inflammation as early signs of atherosclerosis in long-term survivors of disseminated testicular cancer. Eur J Cancer 2004; 40: 701-6.

34. Thogersen AM, Jansson JH, Boman K, Nilsson TK, Weinehall L, Huhtasaari F, et al. High plasminogen activator inhibitor and tissue plasminogen activato levels in plasma precede a first acute myocardial infarction in both men and women: evidence for fibrinolytic system as an independent primary risk factor. Circulation 1998; 98: 2241-7.

35. Juhan-Vague I, Pyke SD, Alessi MC, Jespersen J, Haverkate F, Thompson SG. Fibrinolytic factors and the risk of myocardial infarction or sudden death in patients with angina pectoris. ECAT Study Group. European Concerted Action on Thrombosis and Disabilities. Circulation 1996; 94: 2057-63.

36. Ridker PM, Cushman M, Stampfer MJ, Tracy RP, Hennekens $\mathrm{CH}$. Inflammation, aspirin, and the risk of cardiovascular disease in apparently healthy men. N Engl J Med 1997; 336: 973-9.

37. Danesh J, Wheeler JG, Hirschfield GM, Eda S, Eiriksdottir G, Rumley A, et al. C-reactive protein and other circulating markers of inflammation in the prediction of coronary heart disease. N Engl J Med 2004; 350: 1387-97.

38. Calabro P, Golia E, Yeh ET. CRP and the risk of atherosclerotic events. Semin Immunopathol 2009; 31: 79-94.

39. Sellmayer A, Limmert T, Hoffmann U. High sensitivity C-reactive protein in cardiovascular risk assessment. CRP mania or usefull screening? Int Angiol 2003; 22: 15-23

40. Nuver J, Smit AJ, van der Meer J, van den Berg MP, van den Graaf WTA Meinardi MT, et al. Acute chemotherapy-induced cardiovascular changes in patients with testicular cancer. J Clin Oncol 2005; 23: 9130-7.

41. Gietema JA, Meinardi MT, Messerschmidt J, Gelevert T, Alt F, Uges DR, et al. Circulating plasma platinum more than 10 years after cisplatin treatment for testicular cancer. Lancet 2000; 355: 1075-6.
42. Efstathiou E, Logothetis $\mathrm{CJ}$. Review of late complications of treatment and late relapse in testicular cancer. J Natl Compr Canc Netw 2006; 4: 1059-70.

43. Sprauten $\mathrm{M}$, Darrah $\mathrm{TH}$, Peterson $\mathrm{DR}$, Campbell ME, Hannigan RE, Cvancarova $\mathrm{M}$, et al. Impact of long-term serum platinum concentrations on neuro- and ototoxicity in cisplatin-treated survivors of testicular cancer. J Clin Oncol 2012; 30: 300-7.

44. Verheij M, Dewit LG, Valdes Olmos RA, Arisz L. Evidence for a renovascular component in hypertensive patients with late radiation nephropathy. Int $J$ Radiat Oncol Biol Phys 1994; 30: 677-83.

45. Kim TH, Somerville PJ, Freeman CR. Unilateral radiation nephropathy - the long-term significance. Int J Radiat Oncol Biol Phys 1984; 10: 2053-9.

46. Cassady JR. Clinical radiation nephropathy. Int J Radiat Oncol Biol Phys 1995; 31: 1249-56.

47. Haugnes HS, Aass N, Fossa SD, Dahl O, Klepp O, Wist EA, et al. Components of the metabolic syndrome in long-term survivors of testicular cancer. Ann Oncol 2007; 18: 241-8.

48. Nuver J, Smit AJ, Wolffenbuttel BHR, Sluiter WJ, Hoekstra HJ, Sleijfer DT, et al. The metabolic syndrome and disturbances in hormone levels in long-term survivors of disseminated testicular cancer. J Clin Oncol 2005; 23: $3718-25$.

49. Gietema JA, Meinardi MT, van der Graff WT, Sleijfer DT. Syndrome $X$ in testicular cancer survivors. Lancet 2001; 357: 228-9.

50. Laaksonen DE, Niskanen L, Punnonen K, Nyyssonen K, Tuomainen TP, Salonen $\mathrm{R}$, et al. Sex hormones, inflammation and the metabolic syndrome: a population-based study. Eur J Endocrinol 2003; 149: 601-8.

51. Nord C, Bjoro T, Ellingsen D, Mykletun A, Dahl O, Klepp O, et al. Gonadal hormones in long-term survivors 10 years after treatment for unilateral testicular cancer. Eur Urol 2003; 44: 322-8.

52. Corn BW, Trock BJ, Goodman RL. Irradiation-related ischemic heart disease. J Clin Oncol 1990; 8: 741-50.

53. Martinou M, Gaya A. Cardiac complications after radical radiotherapy. Semin Oncol 2013; 40: 178-85.

54. Vasiliadis I, Kolovou G, Mikhailidis DP. Cardiotoxicity and cancer therapy. Angiol 2014; 65: 369-71.

55. Celik T, Yuksel C, Demirkol S, Iyisoy A, Ulutin C. Coronary artery disease associated with radiation therapy. Cent Eur J Med 2010; 5: 180-3.

56. Wethal T, Kjekshus J, Roislien J, Ueland T, Andreassen AK, Wergeland R, et al. Treatment-related differences in cardiovascular risk factors in long-term survivors of testicular cancer. J Cancer Surviv 2007; 1: 8-16.

57. Haugnes HS, Bosl GJ, Boer H, Gietema JA, Brydoy M, Oldenburg J, et al. Long-term and late effects of germ cell testicular cancer treatment and implications for follow up. J Clin Oncol 2012; 30: 3752-63. 